\title{
Analyze the Metabolites in Rats Small Bowel by UPLC- Q-TOF/MS after Oral Administration of Icaritin
}

\author{
Jun Jiang ${ }^{1,2}$, Li Cui ${ }^{2}$, Xiaobin Jia,* \\ ${ }^{1}$ College of Pharmacy, Nanjing University of Chinese Medicine, 138 Xianlin Avenue, Nanjing, Jiangsu Province, China \\ ${ }^{2}$ Key Laboratory of New Drug Delivery System of Chinese Material Medica, Jiangsu Provincial Academy of Chinese Medicine, $100{ }^{\#}$ \\ Shizi Road, Nanjing, Jiangsu Province, China \\ *Corresponding author: xiaobinjia_nj@126.com
}

Received August 11, 2014; Revised October 21, 2014; Accepted October 27, 2014

\begin{abstract}
Icaritin (IT) was the main product of intestinal metabolism of main flavonoid compounds in Epimedium. Our previous study of the intestinal metabolites of IT was mainly focused on detecting the metabolites in rat faeces, which did not directly reacted and premeditate the complex environment of small bowel. Therefore, in this paper, the contents of small bowel were collected and analyzed by ultra performance liquid chromatography/quadrupole time of flight mass spectrometry (UPLC-Q-TOF/ MS), and then the main metabolites and metabolic pathways of IT in rats small bowel were explained clearly. Finally, six major metabolites were found in rats small bowel after oral administration of IT, which were $\mathrm{m} / \mathrm{z}$ 367.1187, 383.1125, 545.1678, 559.1448, 561.1610, and 721.1979, respectively. These six metabolites and their chemical structures were firstly discovered and identified by us. The metabolites m/z 559.1448 and 545.1678 were the combinations of m/z 383.1125 and 369.1308 (IT) linked with one glucuronic acid, respectively. In the intestinal contents, the IT prototype had the highest peak areas, which indicated that IT mainly existed in the form of prototype. Meanwhile, our results further confirmed that the bioavailability of IT might be low, and subsequent research would likely focus on improving the biopharmaceutical properties and enhancing the bioavailability of IT. This study was useful and complementary to our previous findings.
\end{abstract}

Keywords: metabolites, small bowel, icaritin, glucuronidation, UPLC-Q-TOF/MS

Cite This Article: Jun Jiang, Li Cui, and Xiaobin Jia, "Analyze the Metabolites in Rats Small Bowel by UPLC-Q-TOF/MS after Oral Administration of Icaritin.” Journal of Food and Nutrition Research, vol. 2, no. 11 (2014): 819-823. doi: 10.12691/jfnr-2-11-10.

\section{Introduction}

Herbal Epimedium has been commonly used in traditional Chinese medicine for reinforcing the kidney and strengthening the bone [1]. Studies have demonstrated that Epimedium consist of seven major flavonoid compounds, which share a common stem nuclear similar to their parent aglycone icaritin (IT) [2]. Modern pharmacology studies found that IT exerted many pharmacological activities, including antirheumatic [3], anti-osteoporosis [4,5,6], and antitumor [7]. Our recent study on the intestinal metabolism of Epimedium flavonoids had shown that IT was the main product of intestinal metabolism of all seven flavonoid compounds under the effect of intestinal enzyme and flora [8]. However, the research methods was incubation flavonoids at $37^{\circ} \mathrm{C}$ with rat intestinal enzyme and intestinal flora in vitro, which could not react the real complexities of IT in vivo. In small bowel, there were lots of intestinal floras and hydrolytic enzymes $[9,10,11]$, which could promote IT to be furtherly metabolized into various metabolites.

Our previous study also found that IT could be quickly absorbed into blood circulation and reached the maximum plasma concentration after $15 \mathrm{~min}$, and then rapidly conversed to its glucuronidation metabolites after $30 \mathrm{~min}$ $[12,13]$. Meanwhile, IT was mainly excreted through the bile in the form of glucuronidation (m/z 545.1682 and 545.1661). However, the main metabolites of IT in rats feces were oxygenase, dehydrogenation, and IT prototype, in which there was no glucuronidated products been found. Therefore, a series of complex metabolic changes must have occured in the small intestine.

Because of plenty of glucuronidated products existed in the plasma and bile of rats, we believe that the glucuronidated products should be detected in the intestines of rats. Therefore, in order to clearly explained the metabolic pathways and metabolites of IT in intestine, the contents in small bowel were collected and analyzed by ultra performance liquid chromatography/quadrupole time of flight mass spectrometry (UPLC-Q- TOF/MS) in this paper.

Ultimately, 6 main metabolites of IT in rats intestine were detected and resolved by UPLC-Q-TOF/MS, including 4 glucuronidated products and 2 oxidation products. This study complemented our previous research work, and provided important datas for the biopharmaceutical research and drug development of IT.

\section{Materials and Methods}




\subsection{Materials}

IT (purity >98.3\%) was prepared in our laboratory. HPLC-grade acetonitrile, ethanol and methanol were purchased from Merck (Darmstadt, Germany). MS-grade formic acid was obtained from Nanjing Chemical Reagent Co., Ltd (Nanjing, China). Ultrapure water was purified by the Milli-Q water purification system (Millipore, Bedford, MA, USA). All the other reagents and chemicals were analytical grade.

\subsection{Animals and Drug Administration}

Animal experiments were carried out according to the Guide for the Humane Use and Care of Laboratory Animals and were approved by the Animal Ethics Committee of Jiangsu Provincial Academy of Chinese Medicine. Sprague-Dawley rats (SLAC Lab Animal Center, Shanghai, China), with the body weight of $250 \pm$ $20 \mathrm{~g}$, were allowed to acclimatize for 7 days before the experiment. Animals were kept in cages with a standard laboratory diet and tap water under climate-controlled conditions $\left(25^{\circ} \mathrm{C}, 55 \%\right.$ humidity, and $12 \mathrm{~h}$ of light alternating with $12 \mathrm{~h}$ of darkness).

Twelve hours before the experiment, the rats were fasted overnight and only provided deionized water. The rats were divided into 2 groups: IT group and blank control group. Each group contained 6 rats. IT preparation containing $5 \%$ sodium carboxymethyl cellulose [14] were orally administered to IT groups at a single dose of 80 $\mathrm{mg} / \mathrm{kg}$ body weight [15] while blank control group was administered with sodium carboxymethyl cellulose.

\subsection{Samples Collection and Preparation}

Rats were sacrificed after 10 hours [13], and then abdominal cavity was opened along the midline. Firstly, find out the entrance of the bile duct in the small bowel. Secondly, cut off the intestine at $2 \mathrm{~cm}$ from the distal of the bile duct entrance. Thirdly, along the intestines have been cut down to find the cecum, an then cut off the whole small bowel at $2 \mathrm{~cm}$ from the distal of the cecum. Eventually, the intestinal tissues were homogenized, and extracted by ultrasonic wave with $10 \mathrm{~mL}$ ethanol for 30 minutes. Immediately, the extracted solution were refrigerated centrifugation at $12000 \mathrm{r} / \mathrm{min}$ and $-20^{\circ} \mathrm{C}$ for $30 \mathrm{~min}$. The supernatants were transferred out and filtrated $0.22 \mu \mathrm{m}$ micron microporous membrane before being injected and analyzed by UPLC-Q-TOF /MS.

\subsection{Instrumentation and Conditions of UPLC-Q-TOF/MS.}

UPLC-QTOF-MS/MS, a powerful hyphenated technique $[16,17]$, was used for profiling studies of the metabolites of the active ingredients with high resolution, high sensitivity $[18,19,20,21]$. Chromatography was performed using an ACQUITY UPLC system (Waters Corp., Milford, MA, USA) equipped with a conditioned autosampler at $4^{\circ} \mathrm{C}$. The separation was carried out on an Acquity UPLC BEH C 18 column (ID, $1.7 \mu \mathrm{m} ; 2.1 \times 50$ mm; Waters, Milford, MA, USA). The column temperature was maintained at $30^{\circ} \mathrm{C}$. The mobile phase consisted of $0.1 \%$ formic acid water (solvent $\mathrm{A}$ ) and acetonitrile (solvent $\mathrm{B}$ ). The linear gradient elution program of IT was as follows: 0-3 min, 5-50\% B; 3-6 min, 50-100\% B; 6-9 min, $100 \%$ B; 9-10 min, $100-5 \%$ B; $10-$ $11 \mathrm{~min}, 5 \% \mathrm{~B}$. The injection volume was $5 \mu \mathrm{L}$.

The high mass resolution experiments were performed on a Synapt Q-TOF mass spectrometer (Waters, Milford, MA) equipped with an ESI source under the positive ion mode. The capillary and cone voltages were 2000 and 40 $\mathrm{V}$, respectively. The desolvation gas (nitrogen) was set to $700 \mathrm{~L} / \mathrm{h}$ at $350^{\circ} \mathrm{C}$, and the source temperature was $120^{\circ} \mathrm{C}$. Mass range was scanned from 50 to $1200 \mathrm{Da}$ and corrected during acquisition using an external reference (lock spray) consisting of a solution of $600 \mathrm{ng} / \mathrm{mL}$ leucine enkephalin ( $\mathrm{m} / \mathrm{z} 566.2771)$ infused at a rate of $5 \mu \mathrm{L} / \mathrm{min}$. The transfer CE and trap CE to acquire MS date were 4 and $6 \mathrm{eV}$, respectively, whereas the transfer CE was $15 \mathrm{eV}$ and the trap CE was ramped from 25 to $40 \mathrm{eV}$ to acquire MS/MS data. The raw data was acquired and processed by MassLynx (version 4.1; Waters).

\section{Results and Discussion}

In this paper, our study demonstrated that six major metabolites of IT were found except its prototype. There were $\mathrm{m} / \mathrm{z}$ 367.1187, 383.1125, 545.1678, 559.1448, 561.1610, and 721.1979, respectively. Meanwhile, their contents order was tentatively determined from their peak areas, as follows: $\mathrm{m} / \mathrm{z}$ 369.1308, 545.1678, 561.1610, 721.1979, 559.1448, 367.1187, and 383.1125. These six metabolites and their chemical structures were firstly discovered and identified by us. After carefully analyzing the structures of metabolites, it was found that the metabolites $\mathrm{m} / \mathrm{z} 559.1448$ and 545.1678 were the combinations of $\mathrm{m} / \mathrm{z} 383.1125$ and 369.1308 (IT) linked with one glucuronic acid, respectively.

In the intestinal contents, the IT prototype had the highest peak areas 7892.90, which indicated that IT mainly existed in the form of prototype. According to the value of peak areas, the metabolites $\mathrm{m} / \mathrm{z} 545.1678$ was the second high with the peak areas 3335.50, which was integrated with one glucuronide at the position C-3rd. It was interesting that the metabolites $\mathrm{m} / \mathrm{z} 561.1610$ and 559.1448 were also integrated with one glucuronide at the position C-3rd. The metabolites $\mathrm{m} / \mathrm{z} 721.1979$ was integrated with two glucuronides at C-3rd and C-7th position, respectively. However, the content of $\mathrm{m} / \mathrm{z}$ 721.1979 was lower than others.

Our previous study found that the main metabolites of IT in rats bile were $\mathrm{m} / \mathrm{z} 545.1678$ and 721.1979 [12], which were further excreted into the intestine via the bile duct. Our previous study also found that there was little glucuronide conjugate in rats faeces. This phenomenon indicated that the glucuronide conjugates could be all finally hydrolyzed by a large number of intestinal bacteria and enzymes in intestine, and then excreted through the feces. However, in the metabolic processes throughout the small intestine, there were still plenty of glucuronidation products.

The metabolism of the active substance in small bowel was very complex. The intestinal microflora comprised a complex ecosystem of a large variety of bacteria which could affect the metabolism of the active substance in vivo [11], especially lactase phlorizin hydrolase (LPH). Meanwhile, the main function of LPH enzyme in small 
bowel was to hydrolyze the glucuronidation products Therefore, plenty of the glucuronidation products $\mathrm{m} / \mathrm{z}$ 721.1979, 545.1678, 559.1448, and 561.1610 of IT in the small bowel would be hydrolyzed into their homologous prototype finally, such as $\mathrm{m} / \mathrm{z} 369.1308,383.1125$, or 367.1187. That was why we did not detect out the glucuronidation conjugates of IT in rat feces. This phenomenon was complementary to our previous findings.
The metabolic processes of IT in small intestine was a complex process, and its main metabolic way was hydrolyzed by intestinal bacteria and related enzymes. A large number of IT was excreted in the form of its prototype via feces [8]. These results indicated that the bioavailability of IT might be low, and subsequent research would likely focus on improving the biopharmaceutical properties and enhancing the bioavailability of IT.

\section{Conclusions}

Table 1. Metabolites of IT in osteoporotic rats $(n=3)$ parts permillion, $\mathrm{mg} / \mathrm{L}$.

\begin{tabular}{|c|c|c|c|c|c|}
\hline Metabolites & Formulas & $\mathrm{t}_{\mathrm{R}}(\min )$ & Molecular Weight (m/z) & PPM $(\mu \mathrm{g} / \mathrm{L})$ & Peak areas \\
\hline $\mathrm{P}$ & $\mathrm{C}_{21} \mathrm{H}_{20} \mathrm{O}_{6}$ & 10.12 & 369.1308 & -8.1 & 7892.9 \\
\hline N1 & $\mathrm{C}_{21} \mathrm{H}_{18} \mathrm{O}_{6}$ & 11.38 & 367.1187 & 1.50 & 47.0 \\
\hline N2 & $\mathrm{C}_{21} \mathrm{H}_{18} \mathrm{O}_{7}$ & 9.82 & 383.1125 & -1.40 & 2.5 \\
\hline N3 & $\mathrm{C}_{27} \mathrm{H}_{28} \mathrm{O}_{12}$ & 7.21 & 545.1678 & 3.50 & 3335.5 \\
\hline N4 & $\mathrm{C}_{27} \mathrm{H}_{26} \mathrm{O}_{13}$ & 5.74 & 559.1448 & 0.60 & 58.8 \\
\hline N5 & $\mathrm{C}_{27} \mathrm{H}_{28} \mathrm{O}_{13}$ & 5.76 & 561.1610 & 0.40 & 97.1 \\
\hline N6 & $\mathrm{C}_{33} \mathrm{H}_{36} \mathrm{O}_{18}$ & 5.95 & 721.1979 & -0.10 & 82.7 \\
\hline
\end{tabular}

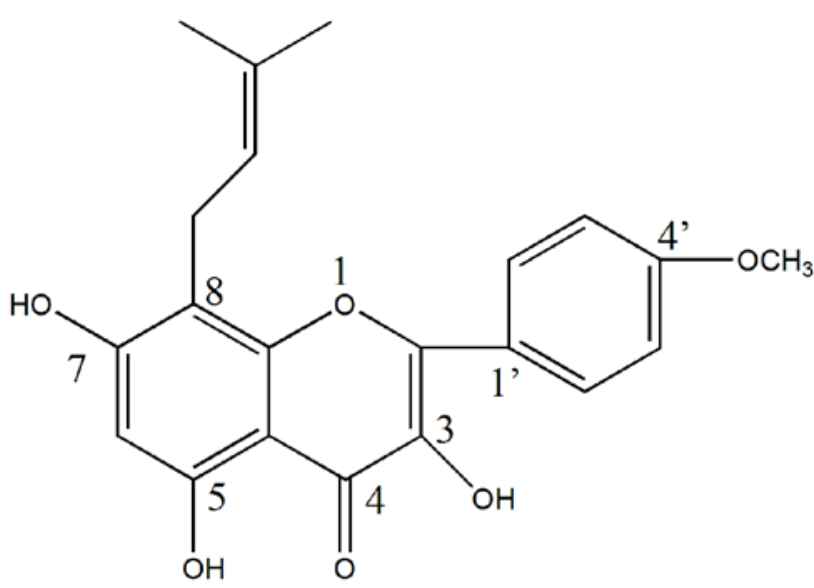

IT

Figure 1. Chemical structures of IT

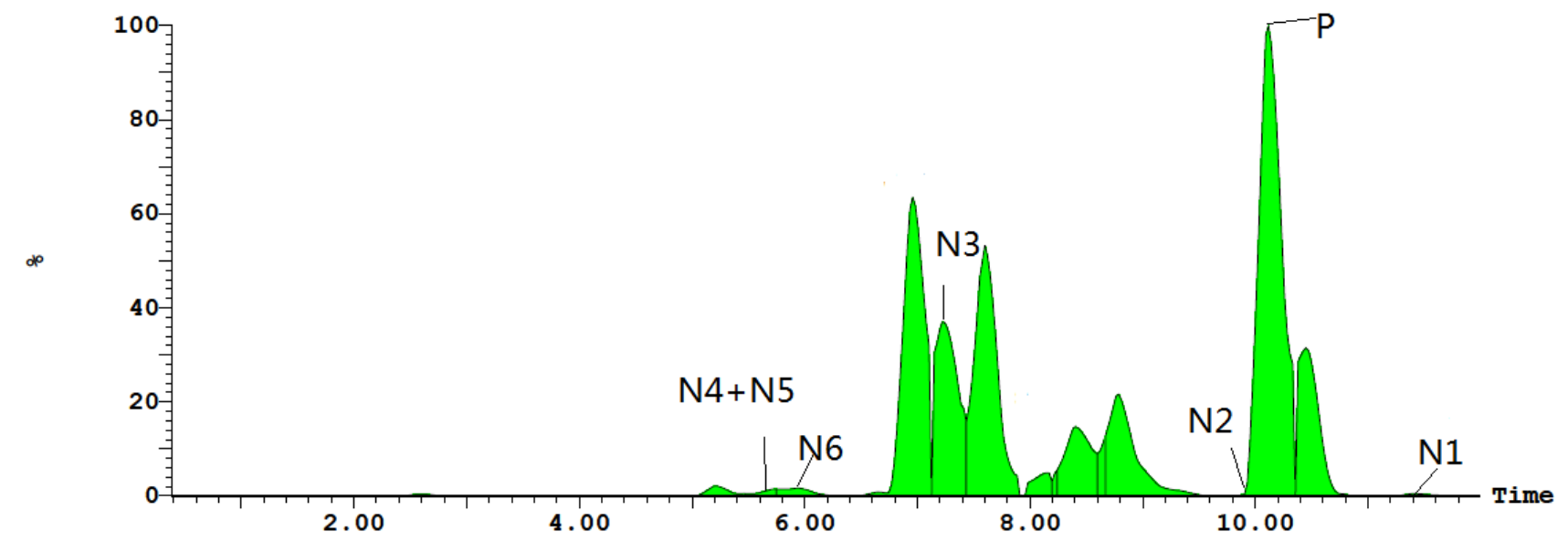

Figure 2. Chromatograms of the metabolites of IT in rats small bowels 

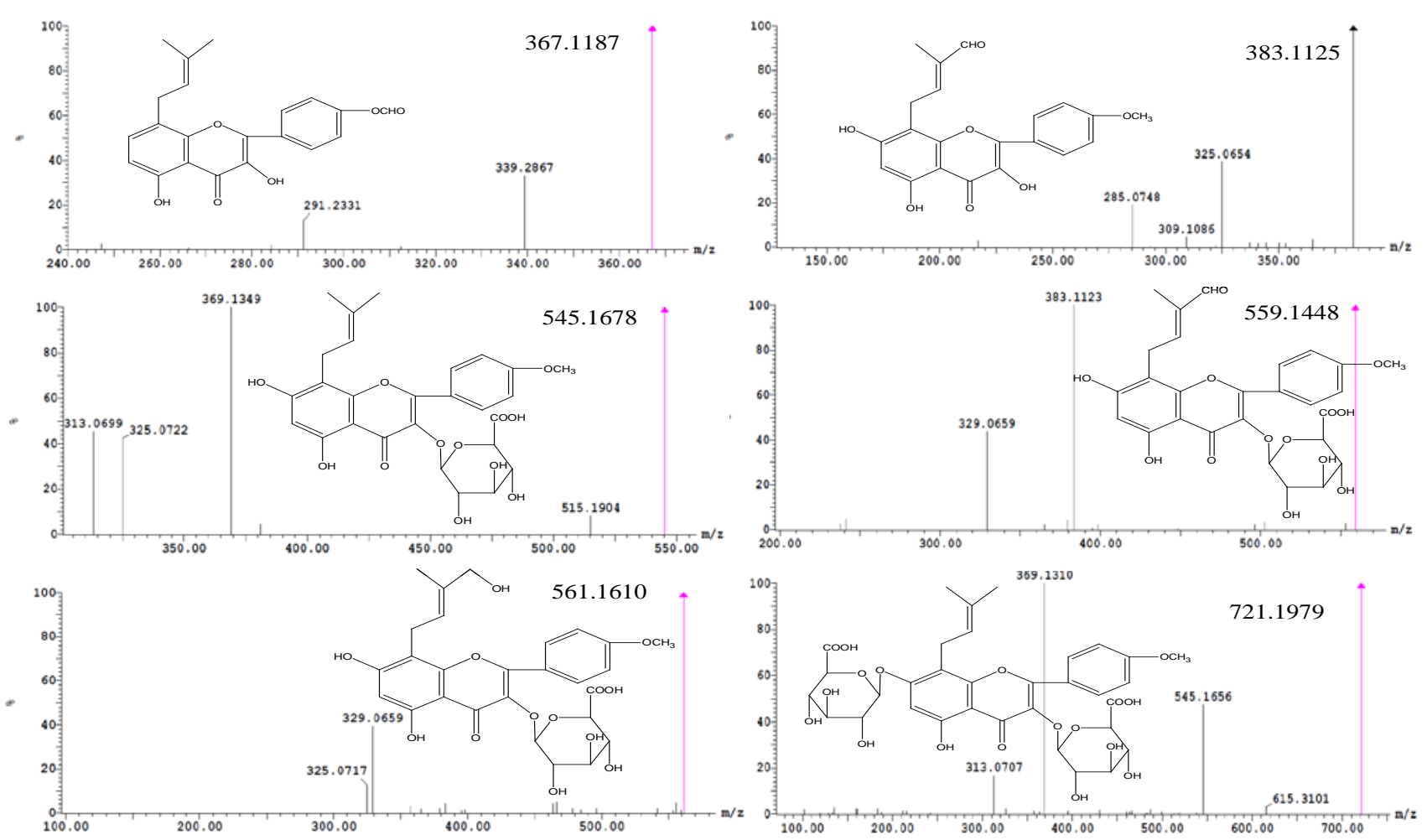

Figure 3. The mass spectral fragmentation of main metabolites of IT in rats small bowels

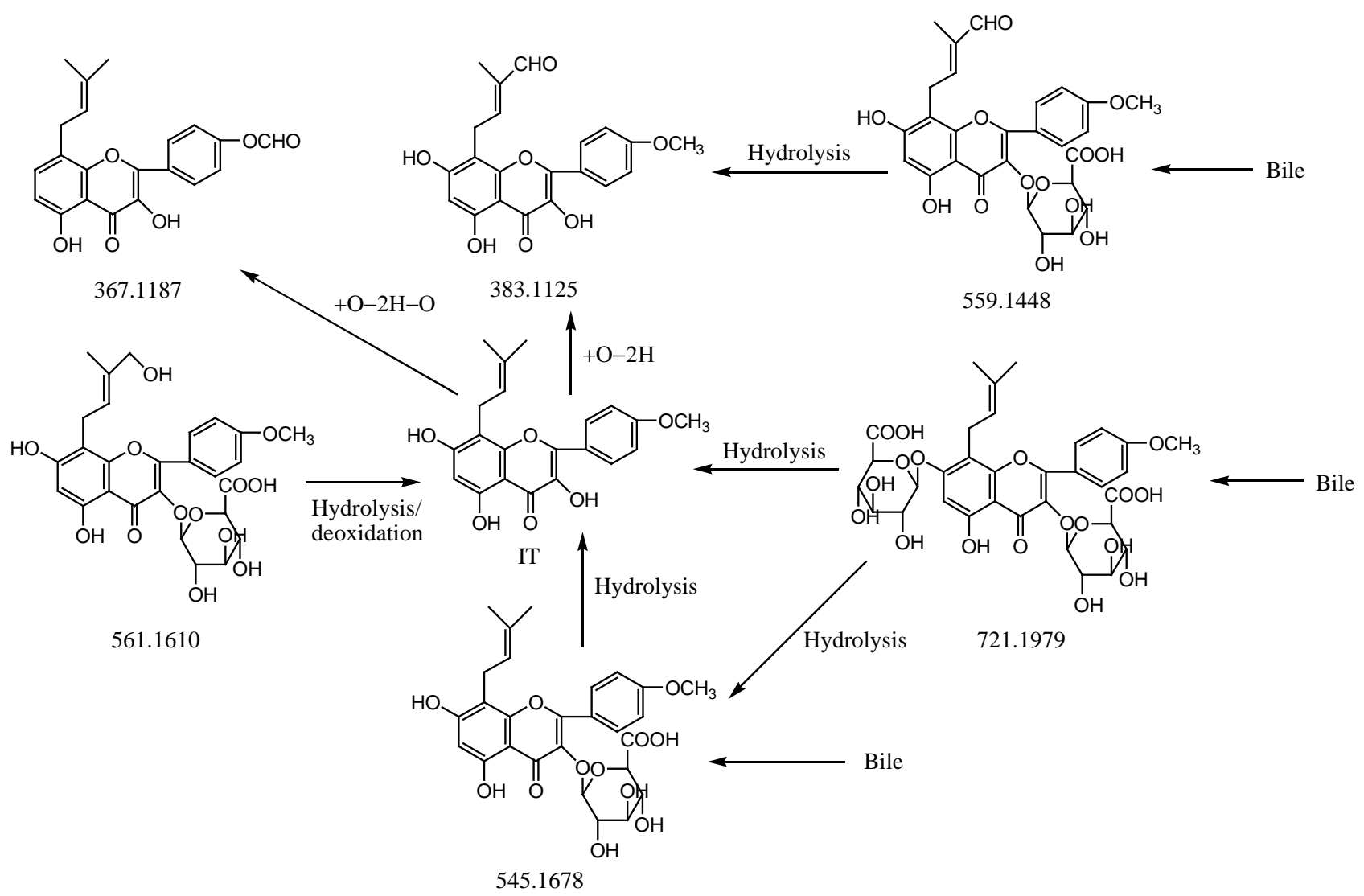

Figure 4. The metabolic pathways of IT a in rats small bowels

A large number of glucuronidation conjugates would be left in the small bowel, which could be further hydrolyzed into their corresponding prototypes by intestinal bacteria and related enzymes. Meanwhile, plenty of IT was excreted in the form of its prototype via feces.

\section{Acknowledgements}

This work was supported by the Natural Science Foundation of China (Nos. 81274088)

\section{References}

[1] Ma, H., He, X., Yang, Y., Li, M., Hao, D., Jia, Z., "The genus Epimedium: an ethnopharmacological and phytochemical review," Journal of Ethnopharmacology, 34 519-541. 2011. 
[2] Lee, S.H., Jung, B.H., Kim, S.Y., Chung, B.C., "Determination of phytoestrogens in traditional medicinal herbs using gas chromatography-mass spectrometry," Journal of Nutritional Biochemistry, 15 452-460. 2004.

[3] Cui, L., Sun, E., Zhang, Z.H., Tan, X.B., Wei, Y.J., Jin, X., Xiao, B.J., "Enhancement of epimedium fried with suet oil based on in vivo formation of self-assembled flavonoid compound nanomicelles,” Molecules, 17 12984-12996. 2012.

[4] Zhang, J.F., Li, G., Meng, C.L., Dong, Q., Chan, C.Y., He, M.L., "Total flavonoids of Herba Epimedii improves osteogenesis and inhibits osteoclastogenesis of human mesenchymal stem cells," Phytomedicine, 16 521-529. 2009.

[5] Zhang, D., Zhang, J., Fong, C., Yao, X., Yang, M., "Herba epimedii flavonoids suppress osteoclastic differentiation and bone resorption by inducing G2/M arrest and apoptosis,” Biochimie, 94 2514-2522. 2012.

[6] Zhang, G., Qin, L., Hung, W.Y., Shi, Y.Y., Leung, P.C., Yeung, H.Y., "Flavonoids derived from herbal Epimedium Brevicornum Maxim prevent OVX-induced osteoporosis in rats independent of its enhancement in intestinal calcium absorption,” Bone, 38 818825. 2006.

[7] Tong, J.S., Zhang, Q.H., Huang, X., Fu, X.Q., Qi, S.T., Wang, Y.P., Hou, Y., Sheng, J., Sun, Q.Y., "Icaritin causes sustained ERK1/2 activation and induces apoptosis in human endometrial cancer cells,” PLoS One, 6 e16781. 2011.

[8] Zhou, J., Chen, Y., Wang, Y., Gao, X., Qu, D., Liu, C., “A comparative study on the metabolism of Epimedium koreanum Nakai-prenylated flavonoids in rats by an intestinal enzyme (lactase phlorizin hydrolase) and intestinal flora,” Molecules, 19 177-203. 2013.

[9] Van, E.J., Robben, J., De, P.G., Merckx, R., Eyssen, H., "Isolation and identification of intestinal steroid-desulfating bacteria from rats and humans," Applied and Environmental Microbiology, 54 2112-2117. 1988

[10] Chen, Y., Wang, J.Y.,Jia, X.B., Tan, X.B., Hu, M., "Rloe of intestinal hydrolase in the absorption of prenylated flavonoids present in Yinyanghuo,” Molecules, 16 1336-1348. 2011.

[11] Bowey, E., Adlercreutz, H., Rowland, I., "Metabolism of isoflavones and lignans by the gut microflora: a study in germ-free and human flora associated rats," Food and Chemical Toxicology, 41 631-636. 2003.

[12] Jiang, J., Feng, L., Sun, E., Li, H., Cui, L., Jia, X., “Metabolic profiling of isomeric aglycones central-icaritin (c-IT) and icaritin (IT) in osteoporotic rats by UPLC-QTOF-MS,” Drug Testing and Analysis. [Epub ahead of print]. 2014.
[13] Chang, Q., Wang, G.N., Li, Y., Zhang, L., You, C., Zheng, Y., "Oral absorption and excretion of icaritin, an aglycone and also active metabolite of prenylflavonoids from the Chinese medicine Herba Epimedii in rats,” Phytomedicine, 19 1024-1028. 2012.

[14] Qian, Q., Li, S.L., Sun, E., Zhang, K.R., Tan, X.B., Wei, Y.J., Fan, H.W., Cui, L., Jia, X.B., "Metabolite profiles of icariin in rat plasma by ultra-fast liquid chromatography coupled to triplequadrupole/time-of-flight mass spectrometry," Journal of Pharmaceutical and Biomedical Analysis, 66 392-398. 2012.

[15] Zhao, H., Fan, M., Fan, L., Sun, J., Guo, D., "Liquid chromatography-tandem mass spectrometry analysis of metabolites in rats after administration of prenylflavonoids from Epimediums," Journal of Chromatography B, 878 1113-1124. 2010.

[16] Liang, Z., Zhang, J., Wong, L., Yi, T., Chen, H., Zhao, Z., Characterization of secondary metabolites from the raphides of calcium oxalate contained in three araceae family plants using laser microdissection and ultra-high performance liquid chromatography quadrupole time of flight-mass spectrometry. European Journal of Mass Spectrometry, 19 195-210. 2013.

[17] Li, S.L. Shen, H., Zhu, L.Y., Xu, J., Jia, X.B., Zhang, H.M., "Ultra-high- performance liquid chromatography quadrupole/time of flight mass spectrometry based chemical profiling approach to rapidly reveal chemical transformation of sulfur-fumigated medicinal herbs, a case study on white ginseng," Journal of Chromatography A, 1231 31-45. 2012.

[18] Gika, H.G., Theodoridis, G.A., Wilson, I.D., "Hydrophilic interaction and reversed-phase ultra-performance liquid chromatography TOFMS for metabono- mic analysis of Zucker rat urine”. Journal of Separation Science, 31 1598-1608. 2008.

[19] He, C., Zhou, D., Li, J., Han, H., Ji, G., Yang, L., "Identification of 20(S)-protopanaxatriol metabolites in rats by ultra-performance liquid chromatography coupled with electrospray ionization quadrupole time-of-flight tandem mass spectrometry and nuclear magnetic resonance spectroscopy,” Journal of Pharmaceutical and Biomedical Analysis, 88 497-508. 2014.

[20] Lenz, E.M., Williams, R.E., Sidaway, J., Smith, B.W., Plumb, R.S., Johnson, K.A., "The application of microbore UPLC/oa TOF-MS and ${ }^{1} \mathrm{H}$ NMR spectroscopy to the metabonomic analysis of rat urine following the intravenous administration of pravastatin,” Journal of Pharmaceutical and Biomedical Analysis, 44 845-852. 2007.

[21] Cui, L., Sun, E., Zhang, Z., Qian, Q., Tan, X., Xu, F., Jia, X.B., "Metabolite profiles of epimedin $\mathrm{B}$ in rats by ultraperformance liquid chromatography quadrupole time of flight mass spectrometry," Journal of Agricultural and Food Chemistry, 61 3589-3599. 2013. 